\title{
Research on Key Technologies of 4G - LTE Uplink Physical Link GUO Yan ${ }^{1, a}$ \\ ${ }^{1}$ Chongqing College of Electronic Engineering, Chongqing 401331, China \\ a76794003@qq.com
}

Keywords: Key Technologies, 4G - LTE, Uplink Physical Link

\begin{abstract}
G-LTE system is a long-term evolution technology proposed by 3GPP. It belongs to quasi-4G technology. It has the features of high rate, wide coverage and low latency. The physical layer is the bottom of the system and the key of technological innovation of mobile communication system. In this paper, some key technologies of the physical layer are studied from the perspective of the physical layer hardware demonstration. The main problems are OFDM synchronization, channel estimation and equalization, multiuser reuse, physical layer algorithm and so on.
\end{abstract}

\section{Introduction}

With the development of multimedia services and Internet technology, wireless communication technology has been put forward higher requirements, is now moving in any place, time, to any person to provide high-speed and reliable communications services to this goal. Mobile communication system has undergone three changes: the first generation of analog communication (1G), the second generation of digital communication (2G) and the third generation (3G) of high-speed wireless broadband network, the current three generations / fourth generation (B3G / 4G) The network has also been a huge development.

Long Term Evolution (LTE) is 3GPP-dominated quasi-4G technology (commonly known as 3.9G), and LTE-Advanced is an enhanced version of LTE technology, support all the features of LTE and backward compatibility, is justifiably 4G technology. This dissertation is devoted to the research of LTE uplink physical layer algorithm implementation, OFDM synchronization technology, channel estimation, PUCCH multi-user transmission and so on.

\section{The 4G-LTE Multiplexing Technology}

In the development of cellular wireless communication technology, the development of multiplexing technology plays a key role. The first generation cellular communication adopts FDMA (Frequency Division Multiple Access), the second generation cellular communication adopts TDMA (Time Division Multiple Access), the third generation cellular communication mainly adopts CDMA (Code Division Multiple Access, Code division multiplexing).

LTE uses Orthogonal Frequency Division Multiplexing (OFDM) technology to reduce the guard band between subcarriers. It divides the whole channel into several mutually orthogonal sub-channels, converting the high speed data signals into parallel low speed sub data streams and transmitting on the low speed sub-channels. In this way, OFDM significantly improves the spectrum utilization, enabling LTE to transmit more data over a limited bandwidth. 4G-LTE using OFDM technology compared to 3G commonly used in the reuse of CDMA technology has the following advantages:

Anti-Multipath Interference Ability is Strong. OFDM data stream for a number of low-speed data stream, the symbol width is longer, and the introduction of cyclic prefix (CP, Cyclic Prefix) protection, can significantly reduce or eliminate inter-symbol interference (ISI, Inter Symbol Interference). 3G wireless communication uses CDMA for high-speed digital time-domain equalizer, while the complexity of CDMA equalizer will increase with the bandwidth of the sharp increase.

It Is Easier to Adopt MIMO (Multiple-Input Multiple-Output, Multi-Input Multiple-Output, or Multiple Receiver Antenna MTMRA): Each subcarrier of OFDM can be regarded as transmitted in flat fading channel and the influence of antenna increase on system complexity is limited. If the 
MIMO technology is used in CDMA, the MIMO receiver and the channel equalization and mixing processing are needed at the receiving end to increase the complexity of the receiver. The complexity of the receiver will change with the increase of the antenna. OFDM uses multiple antenna technology and the complexity of the system is only a linear increase.

It Has Better Bandwidth Scalability: OFDM IFFT used to complete a high-speed data to a number of low-speed data conversion, by adjusting the IFFT size, you can easily change the carrier bandwidth, system complexity increase is not obvious. CDMA need to increase the chip rate or the use of multi-carrier CDMA way to support greater bandwidth, the receiver complexity increased significantly. The former has a strong bandwidth extension and supports multiple carrier bandwidths to facilitate the flexible deployment of the system, which has poor bandwidth scalability.

It Has Frequency Domain Scheduling Flexibility: OFDM frequency domain scheduling particles smaller, only $180 \mathrm{KHz}$, convenient terminal in different time-frequency space for data transmission, access to the frequency scheduling gain. While the CDMA can only carry the carrier-level scheduling of $1.6 \mathrm{MHz}$ width, scheduling flexibility is poor.

Since the OFDM signal is a result of time domain addition of a plurality of mutually orthogonal subcarriers, it has a high peak-to-average ratio. This will put a higher demand for RF signal generator. RF power amplifiers for mobile devices can not meet the transmission requirements of OFDM due to cost constraints and battery life limitations. To this end, the LTE terminal uplink transmission using SC-FDMA (Single-Carrier FDMA, single carrier frequency division multiplexing), and OFDM, SC-FDMA will transmit bandwidth is divided into several high orthogonal subcarrier resources, different Subcarrier resources are allocated to different users for reuse. The difference is that each sub-carrier allocated to the terminal must be continuous, in this way can reduce the signal peak-to-average ratio, to reduce the signal generator requirements, reduce the purpose of transmitting power.

\section{The Technology Advantages of 4G-LTE}

4G-LTE with greater coverage, higher spectral efficiency, faster transmission speed. Its IP is always on-line, perfect QoS mechanism for traffic information service provides an additional advantage.

Single-Cell Network Coverage Is Greater. LTE has a single cell coverage that is not available in cellular radio networks. TD-LTE coverage is related to system resource configuration and CP (Cyclic Prefix). Special subframe configure 0, regular CP, the maximum coverage distance of up to $107.1 \mathrm{~km}$. Common configuration special sub-frame configure 7 , the conventional $\mathrm{CP}$, the maximum coverage distance can reach $21.41 \mathrm{~km}$. FDDLTE, according to its PRACH (Physical Random Access Channel) and CP values, release 8 supports a maximum of $100 \mathrm{~km}$ cell radius. Greater single-cell coverage to facilitate equipment in the population density, traffic layout of the smaller areas of equipment at a lower cost to ensure full coverage of traffic information services, improve service levels to ensure the efficiency of road traffic and traffic safety.

Transfer Is Faster. LTE has more throughput than the previous cellular communication mode and most wireless communication modes: in the $2 * 2$ MIMO, 64QAM modulation mode, 4G-LTE theoretical maximum transmission rate up to 201Mbps, except for signaling and other network management After the overhead, the actual transfer rate is about $140 \mathrm{Mbps}$. In the construction of commercial network, taking into account the networking status and terminal capacity, it is generally considered that 4G-LTE downlink transmission rate of $100 \mathrm{Mbps}$, uplink transmission rate of $50 \mathrm{Mbps}$. The large amount of traffic information, the large number of users and the high timeliness need to distribute large amounts of data quickly. As a result, the 4G-LTE network with faster transmission speed is more suitable for traffic information release service.

IP Is Always Online. LTE is an all-IP network, with IPV6, all the terminals can get a unique IP address. 4G-LTE compared to the previous cellular network with IP always online features: 4G-LTE is an all-IP network, Based on the all-IP network, each device can be found on the Internet any other destination device, to achieve direct application of the two devices. IP always online features for the personalized network of information dissemination service provides the possibility, 
which is used in LTE traffic information dissemination advantages.

It Has Perfect Qos Mechanism. 4G-LTE has perfect QoS mechanism: In order to support a variety of QoS requirements, 4G-LTE in EPS (EvolvedPacketSystem), establish a different bearer, each bearer corresponds to a QoS. According to the QoS characteristics offered by LTE, bearer can be divided into guaranteed minimum bit rate bearer and non guaranteed minimum bit rate bearer.

\section{The Physical Layer Uplink Key Technologies}

The physical layer is the lowest level of the OSI (Open System Interconnect Open System Interconnection) reference model. Although it is at the lowest level, it is the foundation of the whole open system. Its task is to provide a physical link for data transmission . At the physical layer, the data is processed only as raw bits. This layer specifies the physical interface characteristics and communication rules between the network devices, which specify the mechanical, electrical, functional and procedural characteristics required to establish, maintain and dismantle the physical link. Its role is to ensure that the bit stream in the physical channel transmission.

LTE access network protocol can be divided into three levels of structure, the physical layer (PHY) in which the lowest level, the transmission channel for the interface to provide data transmission services for the upper. The Media Access Control (MAC) layer mainly implements scheduling and HARQ related functions, and provides different logical channels to the Radio Link Control (RLC) sublayer of Layer 2. The radio link control (RLC) layer mainly implements ARQ-related functions. Packet Data Convergence Protocol (PDCP) layer to achieve the main header compression and decompression, data encryption. The Radio Resource Control (RRC) layer is primarily responsible for the control and management of the access layer. Non-access layer (NAS) is mainly responsible for providing control and management of the non-access layer. In a wireless communication system, both the uplink and the downlink are required to use a method to distinguish signals of different users, i.e., multiple access techniques. (FDMA), time division multiple access (TDMA), and code division multiple access (CDMA) are more common in wireless communication systems, such as multiple access, multiple access, multiple access, Three kinds of multiple access ways [7]. The LTE air interface utilizes OFDM-based multiple access schemes with a variable system bandwidth (1.4-20 MHz) by varying the number of subcarriers (72-1200) using a subcarrier width of $15 \mathrm{kHz}$. At the same time, according to different application scenarios, LTE supports two different CP (Cyclic Prefix) length of the system configuration, that is, Normal CP and ExtendedCP, their length is about $4.7 \mu$ s and $16.7 \mu$ s. In the specific implementation, according to the different uplink and downlink communications and equipment, LTE, respectively, selected single-carrier SC-FDMA and multi-carrier OFDMA as the uplink and downlink multiple access technology specific implementation.

OFDM Technology. OFDM is a special multicarrier modulation method, which is similar to the basic idea of traditional FDM multi-carrier modulation. OFDM also transforms high-speed data streams into parallel low-speed data streams by serial-parallel conversion, and then allocates them to A plurality of subchannels of different frequencies are transmitted. The OFDM technology utilizes orthogonal subcarriers, and the spectral interleaving of the subcarriers is overlapped, thus avoiding the guard interval in the traditional FDM system and greatly improving the spectral efficiency. Another advantage of OFDM is that it avoids inter-symbol interference (ISI) and inter-carrier interference (ICI) by introducing a cyclic prefix.

Firstly, the transmission signal is channel coded and interleaved, then the interleaved data bits are serialized and converted, and the data are modulated and mapped to the subcarriers of the OFDM symbols. The pilot symbol After inserting the CP into the corresponding subcarrier, the symbols on all the subcarriers are inverse Fourier transformed to generate the time domain symbols, and the parallel symbols are converted into parallel and serial. After inserting the CP in each OFDM symbol, the digital / And up-conversion to the transmission frequency band for signal transmission. Receiver signal processing is the inverse of the transmitter.

SC-FDMA Technology. Although there are many similarities with LTE downlink, LTE physical layer design of the uplink is still facing many challenges. Specific requirements are as 
follows: 1) Different terminals need to transmit orthogonal uplink signals to obtain the minimum intra-cell interference and maximum system capacity. 2) flexibility to support a wider range of data rates, and according to the signal to noise ratio (SINR) adaptively adjust the data rate. 3) Low enough peak to average ratio (PAPR) of the uplink transmit waveform, in order to avoid the terminal of the power amplifier design trouble, such as cost, size and power consumption. 4) Take full advantage of the wideband channel (maximum 20MHz) brought about by the frequency diversity gain, even if the low-rate data transmission can meet this feature. 5) Supports frequency selective scheduling. 6) support for advanced multi-antenna technology, the use of space diversity and enhance the uplink capacity [6]. Taking into account the full compatibility of uplink and downlink, LTE uplink using orthogonal frequency division multiple access (Orthogonal Frequency Division Multiple Access, OFDMA) program is also a very attractive option. In principle, an OFDMA scheme similar to the downlink satisfies all the upstream design requirements in addition to the low PAPR. SC-FDMA combines the ideal features of OFDM with the low PAPR of single carrier transmission. OFDM is to modulate the data symbols to each subcarrier independently (so that the amplitude of each subcarrier depends on the constellation point position of the corresponding data symbol at the same time), and the modulation symbol on each subcarrier of SC-FDMA is the same time at all subcarriers A linear combination of transmission data symbols. Thus, for each SC-FDMA symbol period, all SC-FDMA subcarriers carry the components of each data symbol, such that SF-FDMA has significant characteristics of single-carrier systems that are much lower than the PAPR of multi-carrier systems such as OFDM . Both OFDMA and SC-FDMA schemes use the frequency domain as a new and flexible resource for the system.

\section{Conclusion}

With the gradual completion of China Mobile 4G-LTE scale network test, the large-scale deployment of 4G-LTE network is imminent, which will generate hundreds of billions of industry results for the entire communication industry. In addition, 4G-LTE network all-IP system architecture and the characteristics of flat network structure will make it more easily achieve the communication with the Internet. The characteristics of 4G-LTE such as high-speed data transmission and large-scale coverage will have a huge impact on our lives. Physical layer is the bottom of the system, but also the most important research direction.

\section{References}

[1] Huifang Zhou: Physical Channels and Modulation, Vol. 6 (2004) No 53, p.25-26

[2] Hongli Zhang: Computer Education, Vol. 12 (2005) No 27, p.74-76

[3] Qin Guo: Physical Channels and Modulation, Vol. 1 (2006) No 33, p.11-14

[4] Jieming Liu: Guangxi Normal University, Vol. 3 (2007) No33, p.121-124

[5] Information on .http://lawbooks.com.cn/lw/lw_view.asp?no=958. 